\title{
Research Article Delay Optimization in Cooperative Relaying with Cyclic Delay Diversity
}

\author{
Slimane Ben Slimane, Bo Zhou, and Xuesong Li \\ Radio Communication Systems, Department of Communication Systems, The Royal Institute of Technology (KTH), \\ Electrum 418, 16440 KISTA, Sweden \\ Correspondence should be addressed to Slimane Ben Slimane, slimane@radio.kth.se
}

Received 1 November 2007; Accepted 5 March 2008

Recommended by J. Wang

Cooperative relaying has recently been recognized as an alternative to MIMO in a typical multicellular environment. Inserting random delays at the nonregenerative fixed relays further improve the system performance. However, random delays result in limited performance gain from multipath diversity. In this paper, two promising delay optimization schemes are introduced for a multicellular OFDM system with cooperative relaying with stationary multiple users and fixed relays. Both of the schemes basically aim to take the most advantages of the potential frequency selectivity by inserting predetermined delays at the relays, in order to further improve the system performance (coverage and throughput). Evaluation results for different multipath fading environments show that the system performance with delay optimization increases tremendously compared with the case of random delay.

Copyright (C) 2008 Slimane Ben Slimane et al. This is an open access article distributed under the Creative Commons Attribution License, which permits unrestricted use, distribution, and reproduction in any medium, provided the original work is properly cited.

\section{INTRODUCTION}

A practical method called cooperative communications has been proposed recently in order to approach the theoretical limits of MIMO technology [1]. Mobile units or relays cooperate by sharing their antennas, so as to create a virtual MIMO system [2], thus enabling to exploit diversity and reducing end-to-end path loss [3].

To achieve greater coverage and capacity, relaying has been proved to be a valuable alternative [4-7] for future generations of wireless networks. There are fundamentally two kinds of relays depending upon whether the received signal is only amplified and forwarded or is processed before forwarding, the former is called a nonregenerative relay (amplify-and-forward relay) and the later is called a regenerative relay (decode-and-forward relay). A relay can also be mobile or stationary.

Inserting delays at the relays can make the channel more frequency selective and enhances system performance [8]. These delays can be totally random or can be predetermined. In order to take advantage of the obtained frequency selectivity of the channel, we can either use coded OFDM signalling or single-carrier system with frequency domain equalization [3]. However, the equivalent relay channel will still experience fading dips that may not be resolved by channel coding or equalization. With channel feedback from the mobile unit to the relays, optimal coherent combining of the relayed signals can be obtained and considerable performance improvement can be achieved [6]. However, such an improvement is obtained at the expense of huge feedback information as full channel state information is needed at the different relays.

The aim of this paper is to optimize the cyclic delays in a cooperative OFDM relaying scheme with cyclic delay diversity. Our objective is to improve the coverage and throughput of the system while minimizing the feedback information from the mobile unit to the relay stations. For this purpose, two algorithms are proposed and studied, one is based on the strongest path and the other is based on linear approximation of the channel phase. The obtained results show that both algorithms provide very good performance which make them very promising for future wireless communications.

The paper is organized as follows: Section 2 presents the cellular/relay system model. Section 3 introduces the 


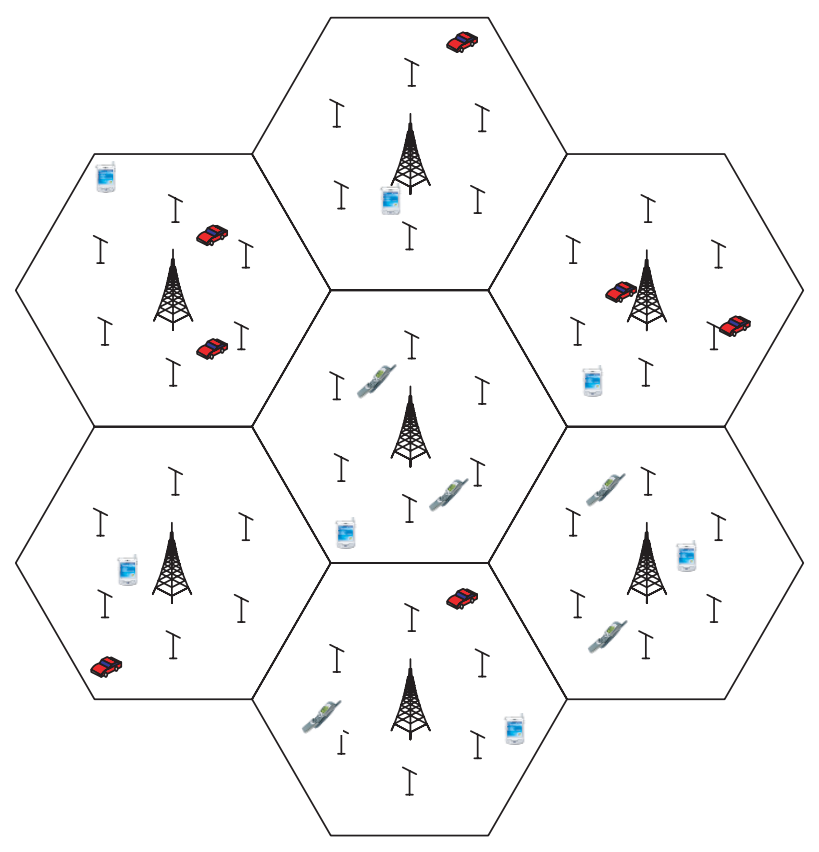

FIGURE 1: System layout of cooperative relaying communication.

delay optimization procedure used in this paper where two different algorithms are given. Section 4 gives a mathematical model of the received signal-to-interference+noise-ratio (SINR) as a function of the number of relays and the different radio channels. Section 5 gives some numerical results to illustrate the behaviour of the algorithms and their performance. Section 6 summarizes the work and provide some suggestions for further studies.

\section{SYSTEM MODEL}

Figure 1 shows the cellular/relay system where each cell consists of a base-station at the center of the cell with omnidirectional antenna and $M$ relays (placed at half the distance from the boundary of the cell). Mobile users are uniformly distributed over the service area. We limit our study to the downlink and assume an OFDM access scheme where the same frequency is used in all the cells (reuse 1).

To better illustrate the system, the communication link within one cell is shown in Figure 2. We assume that the relays operate in a duplex mode where the first time slot is used to receive the OFDM signal from the base station and the second time slot is used to forward a cyclic delayed version (blockwise) of the signal to the mobile unit while the base station is silent. We assume nonregenerative relays where each relay introduces a predetermined cyclic delay, amplifies the signal, and then forwards it. Hence the mobile unit receives two versions of the useful signal that can be combined using maximum ratio combining (MRC) before decoding.

The cyclic delay is usually assumed predetermined or totally arbitrary $[2,3]$. In this paper, we try to identify the delays that can be used at the different relays such that the system throughput is improved.

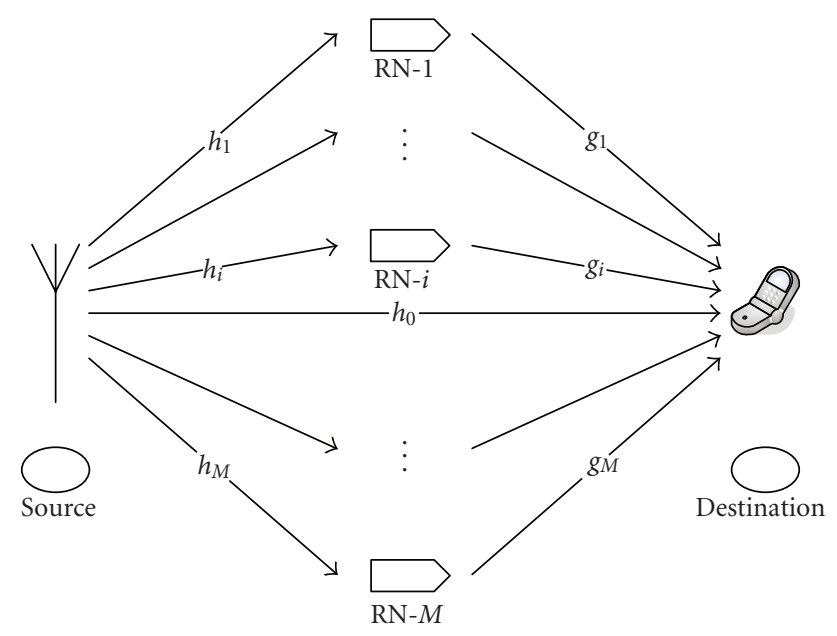

FIgURE 2: Cooperative relaying communication in a single cell.

\section{DELAY OPTIMIZATION ALGORITHM}

Random cyclic delays at the relays do not make the full use of the multipath channel's feature since it increases the frequency selectivity of the radio channel, but does not remove the fading dips. To optimize the delays at a given relay, some information about the channel state between the relay and the mobile unit is needed at the relay. Perfect knowledge of the channel state will provide the best performance, but at the expense of a huge overhead where the channel transfer function at each OFDM subcarrier needs to be sent to the relay [6]. In this paper, we try to reduce this overhead by considering the dominant part of the channel only.

\subsection{Delay optimization based on the strongest path}

Inserting random delay does not make the full use of the multipath channel's feature. An optimal delay allocation approach using coherent combining in large-scale cooperative relaying networks was introduced in [6], but it is well suited for unlimited feedback communications with perfect knowledge of the channel, which is hard to achieve in the practical case. Besides, what we gain from the delay optimization will be lost on the feedback concerning the spectrum efficiency. Although we obtain an optimal delay through this scheme, it comes at expense of the feedback information required.

In this paper, we only take the best segment of the signal from each relay into account and thus only a fractional feedback is required. The benefit of this is with low complexity of the system and high spectrum efficiency; a significant performance gain can be obtained by making the most of the frequency and delay diversity. The idea is locate the strongest path from each relay, cophase it at the relay, and adjust the cyclic delay such that they are in phase and aligned at the mobile unit. This procedure will increase the power of one path of the equivalent relay channel and average the powers of the other paths making the equivalent relay 


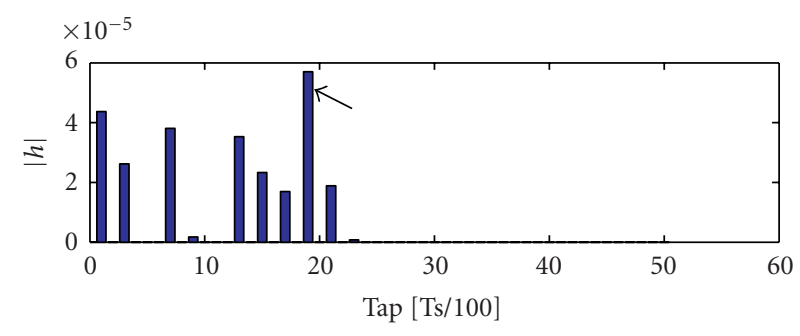

(a)

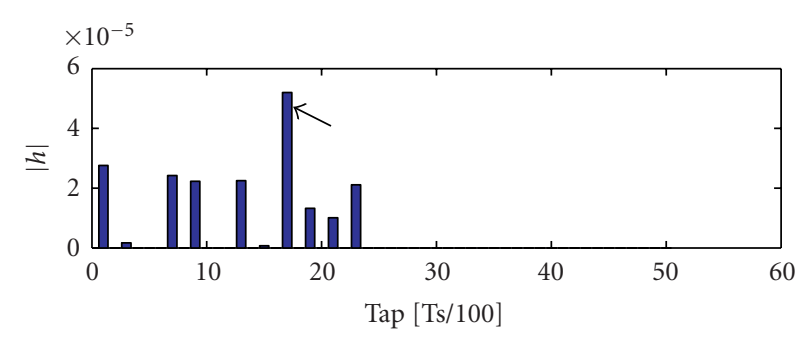

(b)

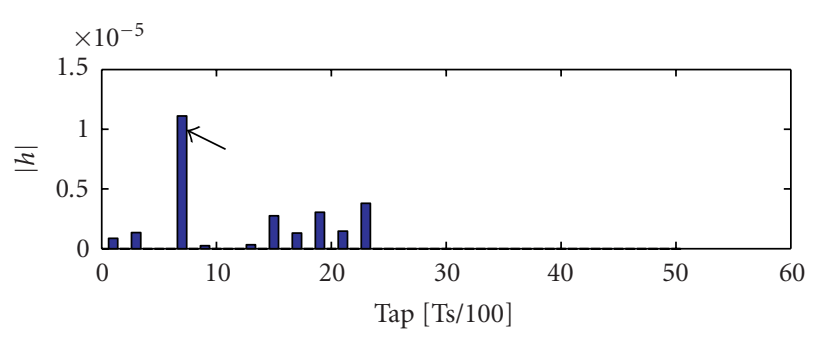

(c)

FIGURE 3: Impulse responses of the initial relay channel $i, i=1,2,3$ in a typical urban environment.

channel appears as Rician fading. Hence each relay requires information about the time delay of the strongest path and its phase only. The benefit of this is a good diversity gain with very limited feedback information.

In order to give a basic introduction to this scheme, let us consider the case of three relay stations with the channel impulse responses shown in Figure 3. The strongest path of each channel is indicated with an arrow.

With the received signals from the relays, the algorithm operates in the following way.

(i) The receiver (MS) locates the strongest path from the three different relays, generates an index of their locations $\left\{l_{1}, l_{2}, l_{3}\right\}$ and phases as $\left\{\theta_{1}, \theta_{2}, \theta_{3}\right\}$, and feed them back to the relays;

(ii) Based on the feedback information about the position of the strongest path and its phase, each relay introduces the proper cyclic shift (optimized delay) to the signal so as the peaks of all signals are aligned at the receiver.

Figure 4 shows how the different channels appear at the receiver side after delay optimization where it is observed that the strongest paths of the different channels are now aligned in time.

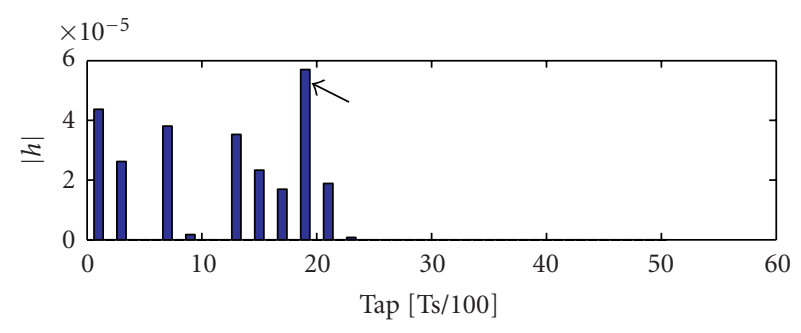

(a)

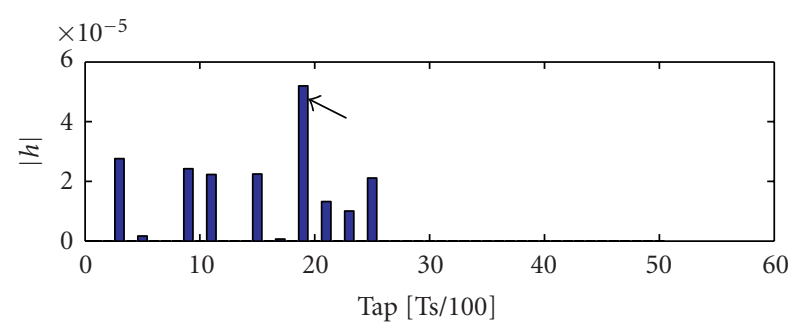

(b)

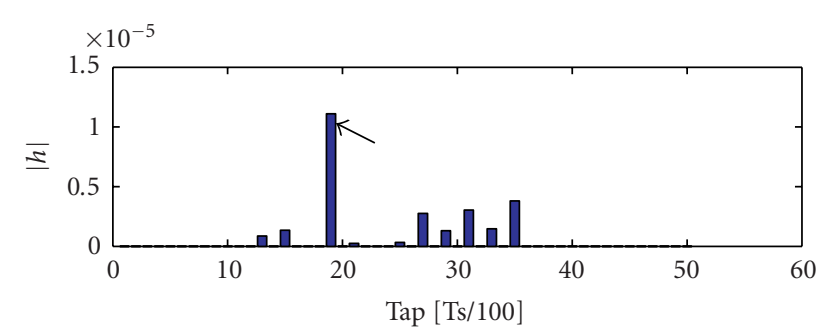

(c)

FIGURE 4: Impulse responses of the three relay channels after delay optimization in a typical urban environment.

(iii) Each relay compensates for the phase of the strongest path such that when the different signals are multiplexed in the air, they will add coherently at the receiver. Hence the total received power of the useful signal will be enhanced. Figure 5 shows the resulting equivalent low pass of the fading multipath relay channel where it is observed that the strongest paths have been added coherently while the secondary paths have been averaged out and kept low values.

\subsection{Linear approximation of the channel phase}

The method discussed in Section 3.1 requires channel state information in the time domain which requires an extra IFFT operation at the mobil unit since channel estimation for OFDM is usually done in the frequency domain. One way to avoid this is by investigating and approximating the channel transfer function phase directly.

Based on the multipath fading channel model, the frequency selective channel can be written as

$$
h(t)=\sum_{k=0}^{K-1} h_{k} \delta\left(t-\tau_{k}\right) .
$$




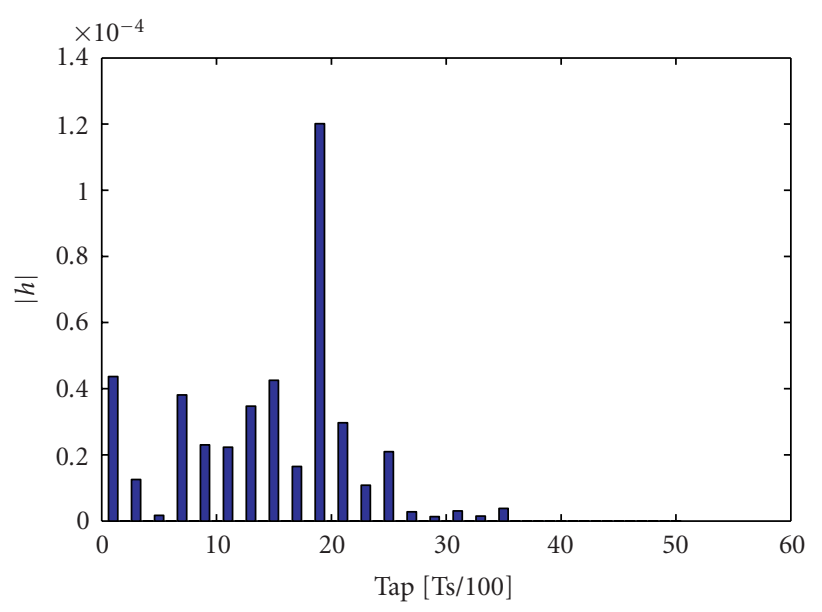

FIGURE 5: Ultimate channel impulse response of the equivalent relay channel.

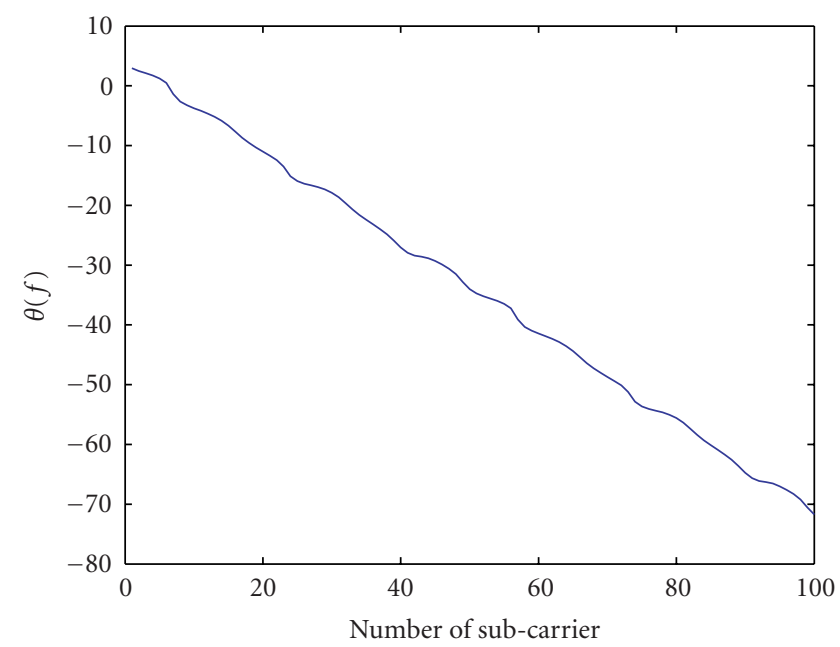

FIgURe 6: Phase variation of multipath fading channel in a typical urban environment with respect to OFDM spectrum.

Assuming $h_{k}$ is slowly varying, the channel transfer function between relay $i$ and the mobile unit can be approximated as

$$
H_{i}(f)=\sum_{k=0}^{K-1} h_{k} e^{-j 2 \pi f \tau_{k}}=\left|H_{i}(f)\right| e^{-j \theta_{i}(f)} .
$$

Figure 6 shows how the $\theta_{i}(f)$ varies with respect to the frequency $f$. From Figure 6 , we notice that the phase $\theta_{i}(f)$ can be approximated as a linear function:

$$
\theta_{i}(f)=\theta_{i}-2 \pi \tau_{i} f,
$$

where $-2 \pi \tau_{i}$ is the slope of the phase and $\theta_{i}$ is a constant phase. Thus the corresponding channel in frequency domain is given by

$$
\begin{aligned}
H_{i}(f) & =\left|H_{i}(f)\right| e^{j \theta_{i}(f)} \\
& \approx\left|H_{i}(f)\right| e^{j\left(-2 \pi f \tau_{i}+\theta_{i}\right)} .
\end{aligned}
$$

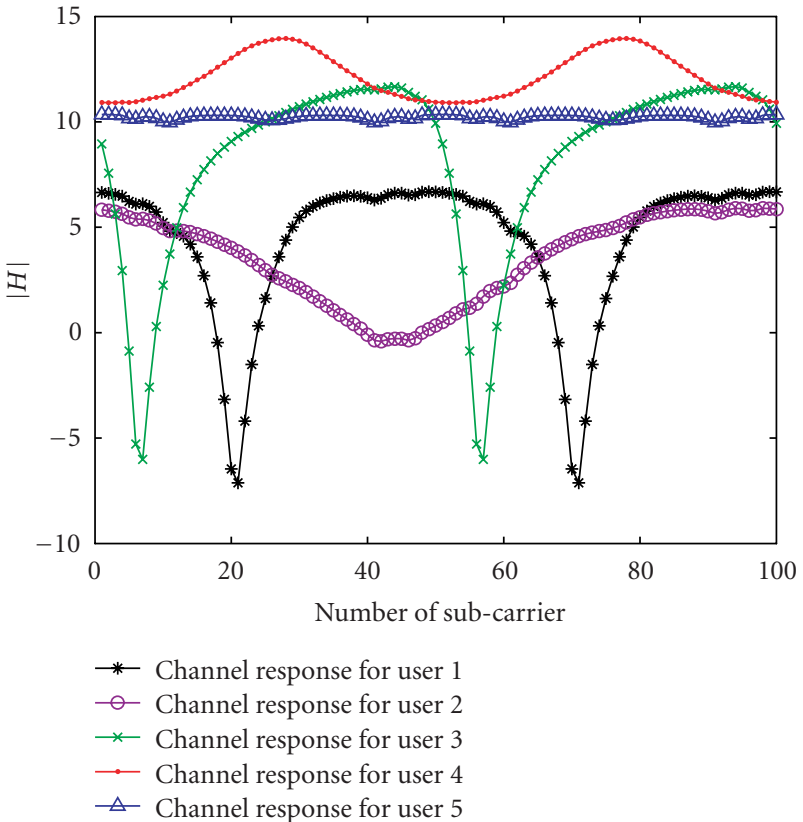

Figure 7: Channel responses in a typical urban environment experienced by 5 different users.

Based on the above formulas, the optimized delay $\tau_{i}$ for relay $i$ can be approximated as

$$
\tau_{i}(f)=-\frac{1}{2 \pi} \frac{d \theta_{i}(f)}{d f} \approx \tau_{i}
$$

Having the estimated delay and the initial phase, each relay channel will make the necessary cophasing and cyclic shifting before signal forwarding. The cyclic delayed signals from the different relays multiplex in the air providing an overall received signal with higher signal amplitude as compared to the case of no delay optimization.

\subsection{Scheduling}

Multiaccess scheme is required to arrange the multiple users sharing the limited resource. In the interest of maximizing the spectrum efficiency thus to limit the cost of the system, which is the main issue from the operators' standpoint [9], an OFDMA scheme with frequency scheduling is considered here.

It should be noted that this scheduling scheme is implemented with priority: one has to give the first priority to the user suffering the most frequency selective channel (with the highest standard deviation) and give second priority to the user having the second highest standard deviation, and so on. This is not the optimal channel allocation algorithm with respect to system throughput, but a fair system from the user's point of view and at the same time the spectrum efficiency remains at a high level.

As illustration of the scheduling scheme, we consider five users per cell. Figure 7 shows the channel frequency response with respect to different users. 
By means of the scheduling scheme presented above, we give higher priority to those users who are not more sensitive to the channel, so as to allocate the subcarriers in a more efficient way.

Applying the scheduling algorithm, we notice from Figure 8 that the users are related well with each other on the spectrum with the help of scheduling.

\section{MATHEMATICAL MODEL}

To model the system, we consider one communication link between the base station and the mobile unit within a given cell. As indicated earlier, the communication is done in two steps: in the first step (first-time slot), the base station transmits information to both mobile unit and the relays and in the second step (second-time slot), the relays forward the information to the mobile unit while the base station is silent.

Considering cell 0 , the received signal at the mobile unit directly from base stations (BS) during the first time slot can be written as

$$
r_{0}(t)=\sum_{i=0}^{N_{c}-1} \sum_{p=0}^{P-1} h_{0, p}^{(i)} s_{i}\left(t-v_{0, p}^{(i)}\right)+z_{0}(t),
$$

where $s_{i}(t)$ is the signal coming from base station $i, h_{0, p}^{(i)}$ and $v_{0, p}^{(i)}$ are the channel attenuation and time delay of path $p$ between base station $i$ and the mobile unit, respectively, $N_{c}$ is the total number of base stations, and $z_{0}(t)$ represents thermal noise.

Assuming that the base stations are synchronized, the demodulated output sample at subcarrier $n$ can be written as

$$
R_{0, n}=H_{0}^{(0)}(n) s_{0, n}+\sum_{i=1}^{N_{c}-1} H_{0}^{(i)}(n) s_{i, n}+Z_{0, n},
$$

where

$$
H_{0}^{(i)}(n)=\sum_{p=0}^{P-1} h_{0, p}^{(i)} e^{-j 2 \pi v_{0, p}^{(i)} n / T}
$$

is the channel transfer function at subcarrier $n, s_{i, n}$ is the received symbol from base station $i$ at subcarrier $n$, and $Z_{0, n}$ is zero-mean complex Gaussian random variable with variance $N_{0}$.

The received signals at the different relays from the base station within cell 0 are given by

$$
\begin{array}{r}
y_{0, m}(t)=\sum_{i=0}^{N_{c}-1} \sum_{p=0}^{P-1} c_{m, p}^{(i)} s_{i}\left(t-v_{m, p}^{(i)}\right)+z_{m}(t), \\
m=0,1, \ldots, M-1 .
\end{array}
$$

Each relay amplifies and retransmits its received signal with the appropriate cyclic delay while the base stations are silent. Hence the received signal at the mobile unit from the different relays during the second time slot can be written as

$$
r_{1}(t)=\sum_{i=0}^{N_{c}-1} \sum_{m=0}^{M-1} \beta_{i, m} \sum_{p=0}^{P-1} g_{m, p}^{(i)} y_{i, m}^{\prime}\left(t-\tau_{m, p}^{(i)}\right)+z_{1}(t),
$$

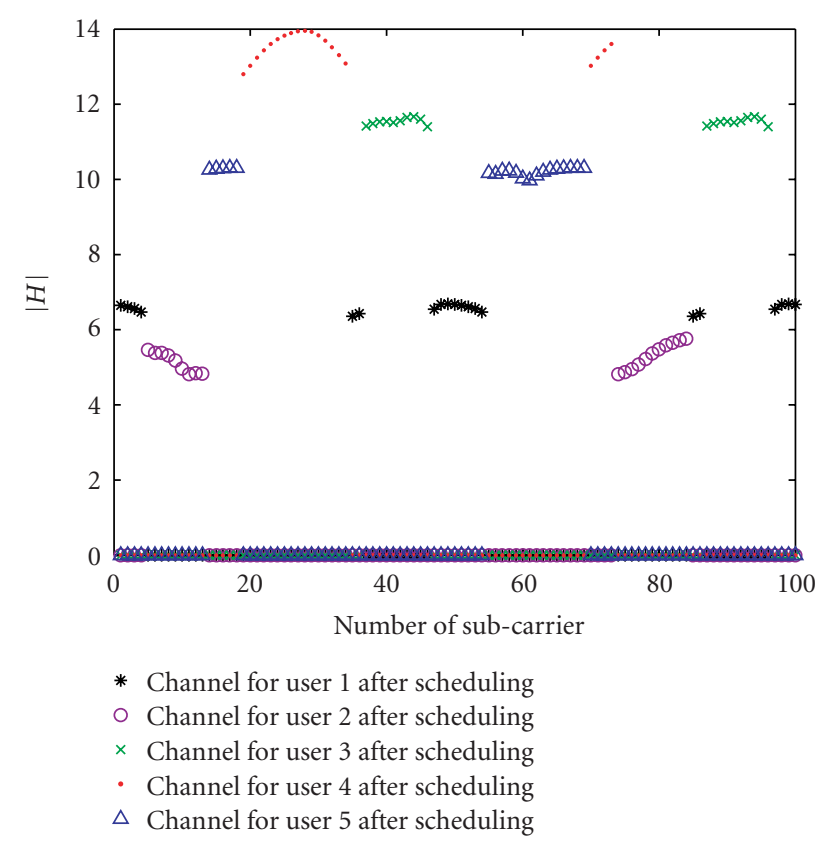

FIgURE 8: Channel allocation to the 5 users after scheduling in a typical urban environment.

where $y^{\prime}(t)$ is the cyclic delay version (blockwise) of $y(t)$ and $\beta_{i, m}$ is the amplification factor used at relay node $m$ within cell $i$ with

$$
\beta_{i, m}=\frac{1}{\sqrt{\sum_{p=0}^{P-1}\left|c_{m, p}^{(i)}\right|^{2}+N_{0} / E_{i}}}
$$

and $E_{i}=p_{i} T$ is the average energy per transmitted symbol of cell $i$.

Assuming that the relays are synchronized, the demodulated signal sample at subcarrier $n$ can be written as

$$
R_{1, n}=H_{1, e}(n) s_{0, n}+\sum_{i=1}^{N_{c}-1} H_{i, e}(n) s_{i, n}+\sum_{i=1}^{N_{c}-1} G_{i, e}(n) s_{i, n}+Z_{1, n}
$$

where

$$
\begin{aligned}
H_{1, e}(n) & =\sum_{m=0}^{M-1} \beta_{0, m} G_{m}^{(0)}(n) C_{m}^{(0)}(n) e^{-j\left(\theta_{0, m}+2 \pi n l_{0, m} / N\right)} \\
H_{i, e}(n) & =\sum_{m=0}^{M-1} \beta_{0, m} G_{m}^{(0)}(n) C_{m}^{(i)}(n) e^{-j\left(\theta_{0, m}+2 \pi n l_{0, m} / N\right)} \\
G_{i, e}(n) & =\sum_{m=0}^{M-1} \beta_{i, m} G_{m}^{(i)}(n) \sum_{k=0}^{N_{c}-1} C_{m}^{(k)}(n) e^{-j\left(\theta_{i, m}+2 \pi n l_{i, m} / N\right)}
\end{aligned}
$$

$G_{m}^{(i)}(n)$ is the channel transfer function between relay $m$ of base station $i$ and the mobile unit at subcarrier $n, C_{m}^{(i)}(n)$ is the channel transfer function between base station $i$ and its relay $m$ at subcarrier $n,\left(l_{i, m}, \theta_{i, m}\right)$ are the optimized cyclic 
TABLE 1: Simulation parameters.

\begin{tabular}{ll}
\hline Parameter & Value \\
\hline Number of subcarriers & 128 \\
Noise floor & $-105 \mathrm{dBm}$ \\
Fast multipath fading & Urban/suburban \\
Path loss exponent $(\alpha)$ & 3.5 \\
Shadow fading standard deviation & $6 \mathrm{~dB}$ \\
Transmit power (base station and relay) & $33 \mathrm{dBm}$ \\
Users per cell & 5 \\
Cell radius & $500 \mathrm{~m} / 1 \mathrm{~km}$ \\
Channels per cell & 20 \\
Number of relays per cell & 6 \\
\hline
\end{tabular}

shift and the phase employed at relay $m$ within cell $i$, and $Z_{1, n}$ is zero-mean complex Gaussian with variance $N_{0}$.

Combining the direct received signal in (7) and that from the relays in (12), the signal-to-interference+noiseratio (SINR) can be written as

$$
\begin{aligned}
\Gamma= & \frac{\left|H_{0}^{(0)}\right|^{2} p_{0}}{\sum_{i=1}^{N_{c}-1}\left|H_{0}^{(i)}\right|^{2} p_{i}+N_{0} W} \\
& +\frac{\left|H_{1, e}\right|^{2} p_{0}}{\sum_{i=1}^{N_{c}-1}\left(\left|H_{i, e}\right|^{2}+\left|G_{i, e}\right|^{2}\right) p_{i}+N_{0} W},
\end{aligned}
$$

where without loss of generality, we have dropped the subcarrier index $n, p_{i}$ is the average transmitted power of signal $s_{i}(t)$, and $W$ is the signal bandwidth.

The throughput is derived from the received SINR using the following expression:

$$
R=C_{B} W \log _{2}\left(1+\frac{\Gamma}{2}\right)
$$

where $C_{B}=1 / 2$ to account for the half duplex operation of the relay node and the factor 2 is to account for practical implementation of channel coding and modulation.

\section{NUMERICAL RESULTS}

Numerical evaluation is performed by system simulation of a two-tier (19 cells) hexagonal cellular system with omnidirectional antenna and 6 relay nodes per cell as illustrated in Figure 1. The proposed algorithms are evaluated by snapshot simulation for the OFDMA system. We assume that users are uniformly distributed over the whole cells. The number of active relays for each user is set to $M=3$. Mobile units are uniformly distributed within the area. The multipath fading channel is modelled as a tapped delay line and based on the models proposed in [10]. A more detailed list of the simulation parameters is given in Table 1 .

With fractional feedback, delay optimization based on strongest path further enhances the channel response compared to inserting random delays at relays. Two different types of channel are considered here: (1) flat fading channel

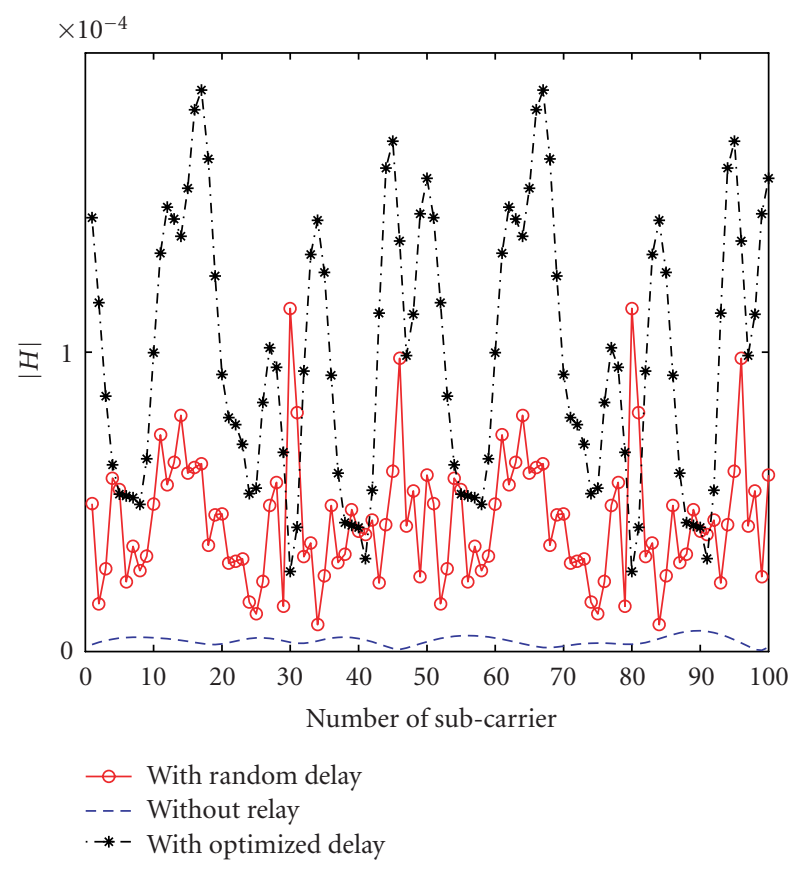

FIGURE 9: A snapshot of a frequency selective channel before and after adding cyclic delays for the case of three active relays.

and (2) frequency selective fading channel. By adding predetermined delays and retransmitting the signal with proper amplification at relay nodes, this delay diversity scheme leads a substantial improvement to the system performance.

By properly selecting the cyclic delay for each relay node, we expect to get a good relay channel that can improve the communication link of the mobile unit. Figure 9 illustrates the channel transfer function of the relay channel with and without cyclic delay diversity for a typical urban environment. It is observed that the initial channel has been improved and the optimized delays have improved the channel gains of the different OFDM subcarriers which make the channel more robust as compared to the case with random delays.

A performance improvement of the OFDMA scheme with frequency scheduling is then expected. As our objective is to assess the performance of the optimized delay scheme, we limit our study to the case of having the same statistical channels between the source and relays, as well as between the relays and the mobile unit. We have investigated the performance of our system in a typical urban and rural area environments [10].

The number of active relays within the cell will affect the received SINR experienced by the user. Figure 10 shows the received SINR at 5 percentile for a given user and with different number of active relays. We notice that having 3 active relays is a good compromise between increased received power and experienced interference.

As we can see from Figure 11, the performance can be improved by increasing the total number of relays per cell, but it can be noted that with more than six relays the system performance has not been improved much. Due to 


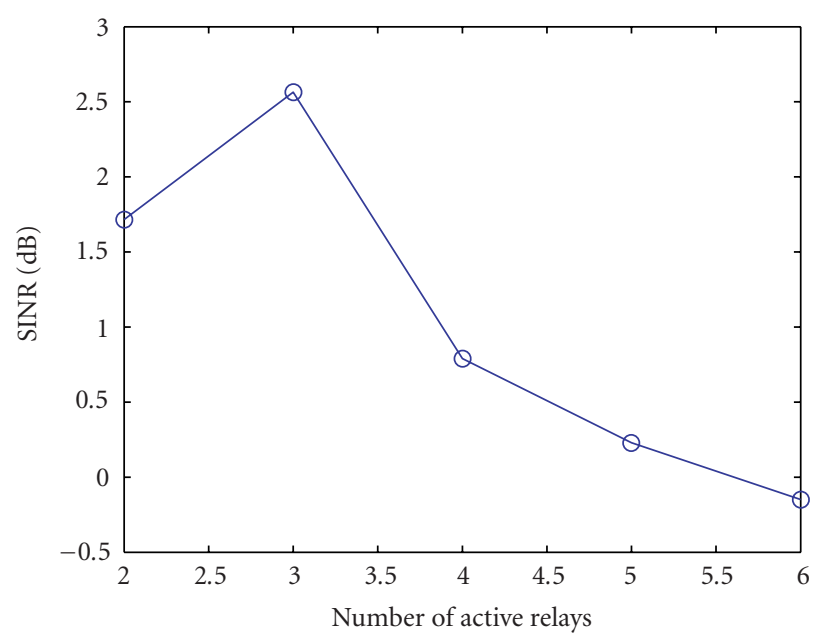

FIgURE 10: The received SINR at 5 percentile with the different number of active relays on an urban environment with a cell radius of $500 \mathrm{~m}$.

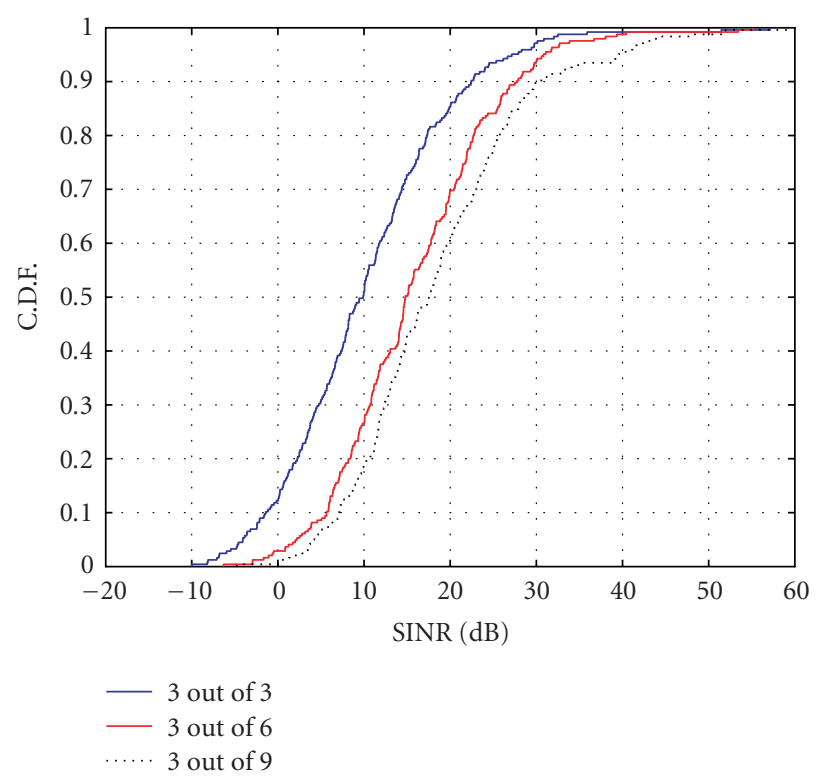

FIGURE 11: Cumulative distribution function of the received SINR with the different number of total relays on an urban environment with a cell radius of $500 \mathrm{~m}$.

the infrastructure cost issue, we considered six relays per cell and we assumed that only three are active at a time. The following simulations are based on this relay selectivity scheme.

Figure 12 shows the cumulative distribution function (CDF) of the combined received SINR with and without delay diversity over an urban environment when the optimized delay is based on the strongest path and with three active relays out of 6 relays. Clearly, the optimized delay algorithm improves the system performance considerably.

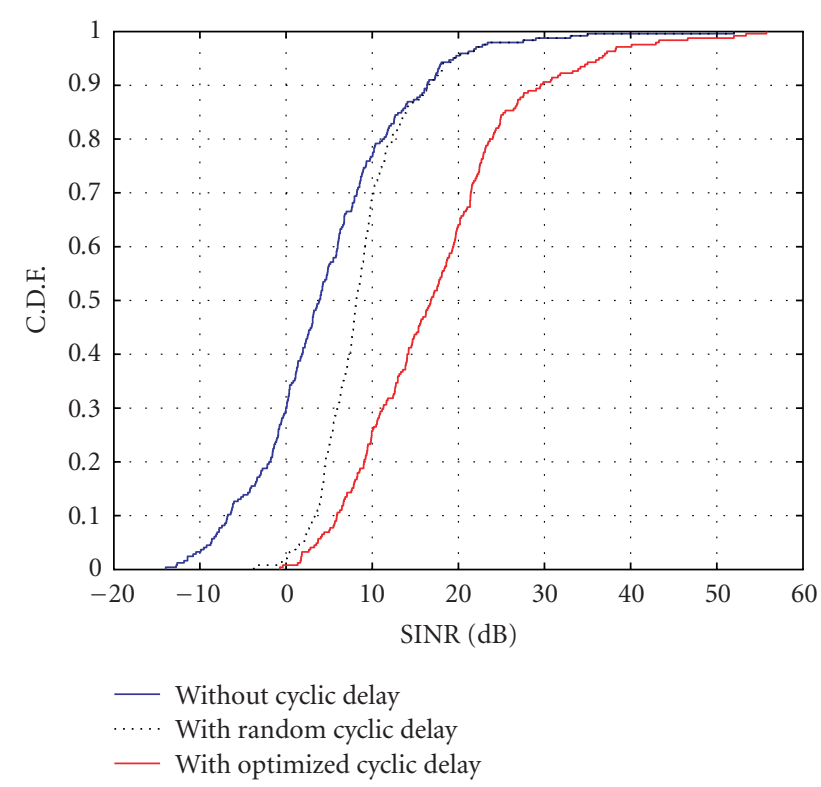

FIGURE 12: Cumulative distribution function of the received SINR on an urban environment with a cell radius of $500 \mathrm{~m}$.

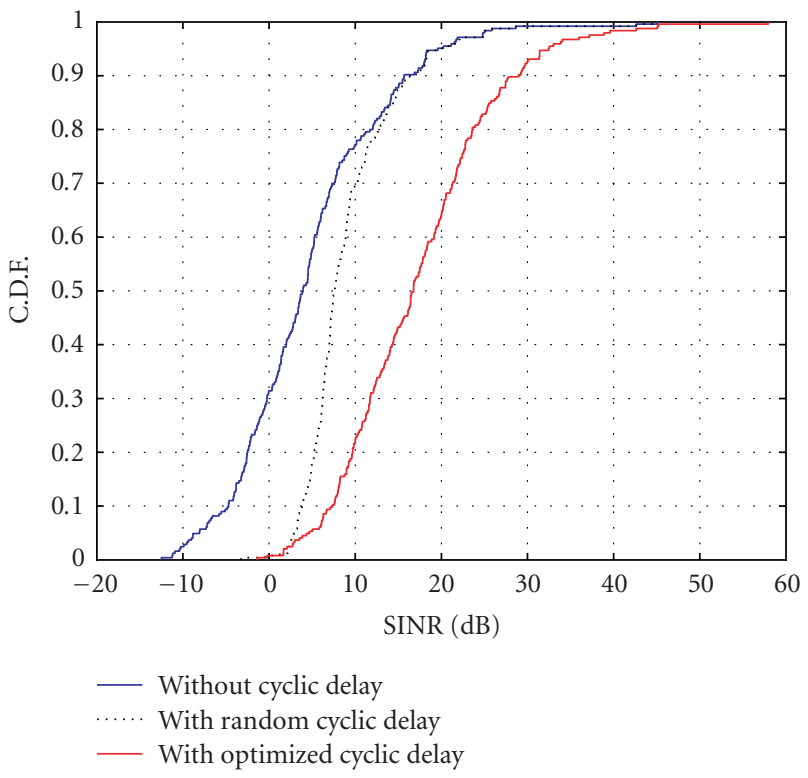

FIGURE 13: Cumulative distribution function of the received SINR on a rural environment with a cell radius of $1000 \mathrm{~m}$.

An improvement of about $3 \mathrm{~dB}$ at 5 percentile of the CDF compared to random delay is obtained.

In a rural environment, we can see that (Figure 13) the system has also been greatly improved by about $3 \mathrm{~dB}$ at 5 percentile of the CDF when introducing optimized delay as compared to the random delay scheme.

Comparing the results of Figures 12 and 13, we notice that when the cell radius increases, the performance of system with optimized delay still remains at a high level. This feature offers us a good solution to guarantee the 


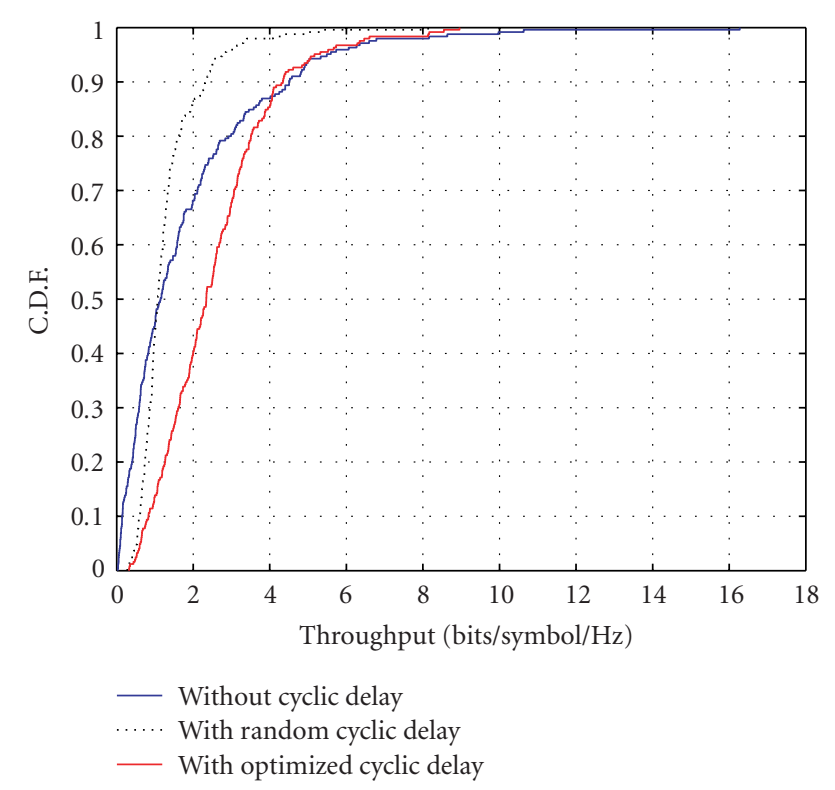

FIgURE 14: Normalized system throughput on an urban environment with a cell radius of $500 \mathrm{~m}$.

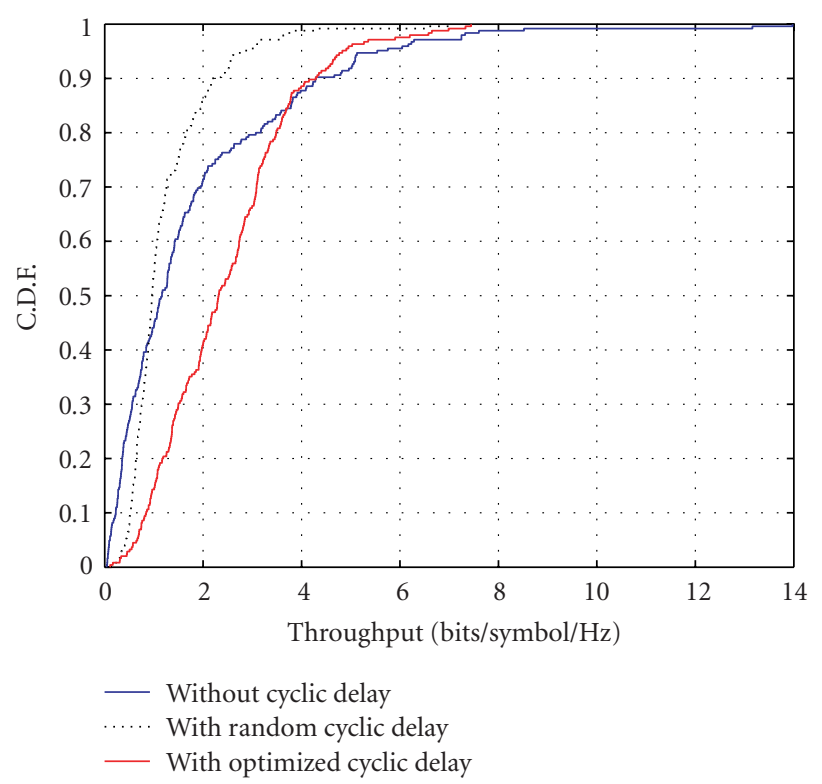

FIGURE 15: Normalized system throughput on a rural environment with a cell radius of $1000 \mathrm{~m}$.

service quality in large coverage case and can reduce the infrastructure cost and at the same time improve the system performance. The system performance is further evaluated in terms of system throughput to support our theoretical derivation. The corresponding normalized throughput for an urban environment has been evaluated and is illustrated in Figure 14 and that on a rural environment is shown in Figure 15. From these simulation results, it is clear that the system throughput increases when using the optimized cyclic delay algorithm on different environments. It is interesting to note that for both environments, relays with random

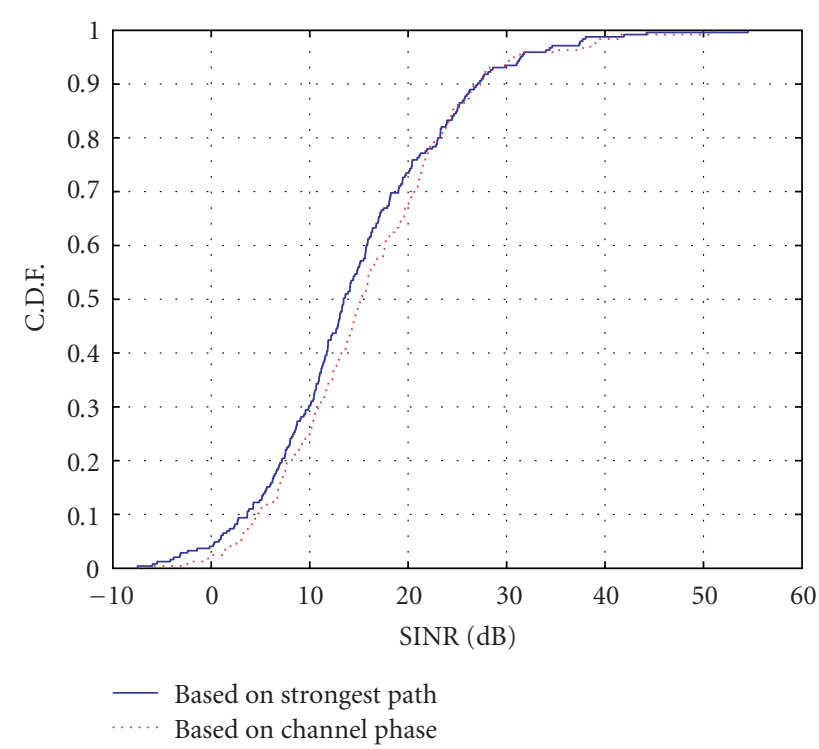

FIGURE 16: Cumulative distribution function of the received SINR for the two optimized algorithms on an urban environment with a cell radius of $500 \mathrm{~m}$.

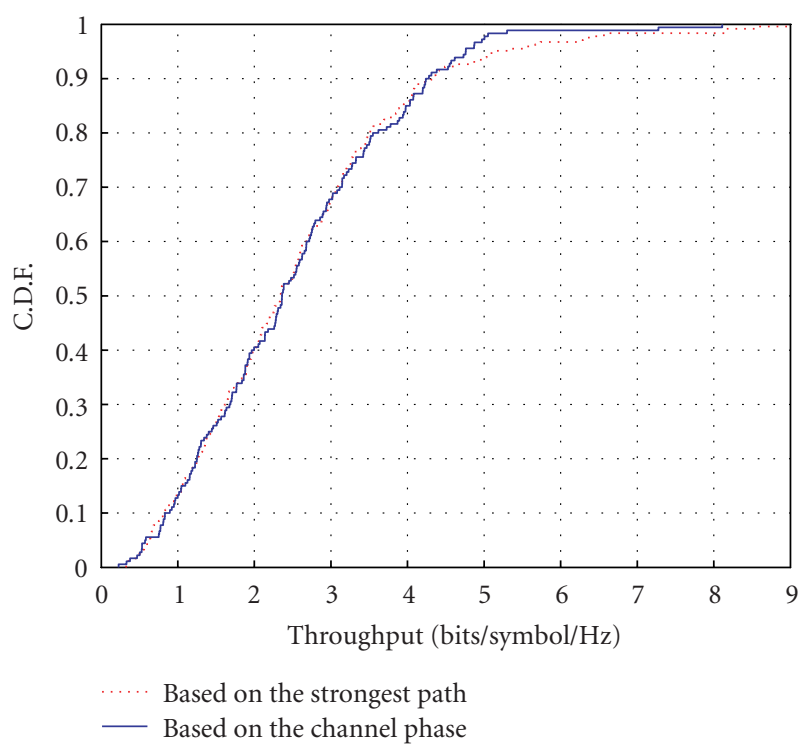

FIgURE 17: Normalized system throughput for the two optimized algorithms on an urban environment with a cell radius of $500 \mathrm{~m}$.

delay do not improve the system throughput in comparison to the case without relay. The crossover in the two curves for random delay and no relay situation occurs due to the interference behaviour. At high coverage, the SINR decreases for random delay compared to the case without relay.

By implementing the two delay optimization schemes proposed in this paper, the corresponding results both in SINR and throughput are shown below. Figure 16 shows the cumulative distribution function of the received SINR for the two optimized algorithms on an urban environment, while Figure 17 shows the normalized throughput. It is observed 
that the two algorithms perform almost in the same way with respect to received SINR as well as throughput. The algorithm based on strongest path performs a little better than the second algorithm. For the linear approximation algorithm, we take an approximate of the slope phase curve (which contains variations) which does not give that accurate optimum delay but gives us a rough idea of how to estimate it. On the other hand, the method based on strongest path tends to give a better approximation of the delay as it adds the paths coherently.

\section{CONCLUSIONS}

In this paper, two promising delay optimization schemes have been proposed based on linear approximation of the channel phase and the strongest path, for a multicellular OFDM system with cooperative relays, in order to take the most advantages of the multipath fading channel by means of exploiting the potential frequency selectivity. The obtained results show that the system performance with delay optimization increases tremendously compared with random delay diversity. Evaluations in different environments further shows that the delay of these optimization schemes is well suited for diverse environments and supports a large coverage. It should be noted that the relays work in a distributed manner and no coordination is needed; besides, both of the delay optimization schemes only require a fractional feedback to substantially improve the system performance. It is quite attractive to the operators who hope to improve the service as well as reduce the system complexity and cost.

We focused in this paper on the delay optimization with limited feedback only relying on the strongest path. One of the interesting points is to investigate how the feedback affects the system performance and what is the optimum degree of feedback with respect to the performance/cost ratio. Implementation of sector antennas will also affect the results by reducing the interference. In addition, the introduction of different scheduling algorithms, for example, always assigning the channel to the user holding the best SINR, could improve the system performance as well. These are some points that can be further explored and studied in the future.

\section{REFERENCES}

[1] F. H. P. Fitzek and M. D. Katz, Cooperation in Wireless Networks: Principles and Applications, Springer, New York, NY, USA, 2006.

[2] A. Nosratinia, T. E. Hunter, and A. Hedayat, "Cooperative communication in wireless networks," IEEE Communications Magazine, vol. 42, no. 10, pp. 74-80, 2004.

[3] S. B. Slimane and A. Osseiran, "Relay communication with delay diversity for future communication systems," in Proceedings of the 64th IEEE Vehicular Technology Conference (VTC '06), pp. 321-325, Montreal, Canada, September 2006.

[4] N. Ahmed, M. A. Khojastepour, and B. Aazhang, "Outage minimization and optimal power control for the fading relay channel," in Proceedings of the IEEE Information Theory
Workshop (ITW'04), pp. 458-462, San Antonio, Tex, USA, October 2004.

[5] J. N. Laneman, D. N. C. Tse, and G. W. Wornell, "Cooperative diversity in wireless networks: efficient protocols and outage behaviour," IEEE Transactions on Information Theory, vol. 50, no. 12, pp. 2062-3080, 2004.

[6] P. Larsson, "Large-scale cooperative relay network with optimal coherent combining under aggregate relay constraints," in Proceedings of the Future Telecommunications Conference, pp. 166-170, Beijing, China, December 2003.

[7] A. Sendonaris, E. Erkip, and B. Aazhang, "User cooperation diversity-part I: system description," IEEE Transactions on Communications, vol. 51, no. 11, pp. 1927-1938, 2003.

[8] A. Yaver, A. Anto, M. U. Khattak, and P. Nagarajan, "Cooperative relaying with cyclic delay diversity," Wireless Networks Course Project, 2006.

[9] J. Zander and S.-L. Kim, Radio Resource Management for Wireless Networks, Artech House, Norwood, Mass, USA, 2001.

[10] GPP TR 25.943 V5.1.0 Technical Report-Release 5, 3rd Generation Partnership Project, June 2002. 